\title{
Elaeagnus Angustifolia extract inhabits cell invasion of human colorectal cancer cells and increases the survival rate of the Drosophila colon cancer model
}

\author{
Arij Fouzat ${ }^{1,3}$, Layla Kamareddine ${ }^{2,3}$, Ala-Eddin Al Moustafa ${ }^{3,4}$, Ashraf Khalil ${ }^{1,3}$
}

${ }^{1}$ College of Pharmacy, ${ }^{2}$ College of Health Sciences, ${ }^{3}$ Biomedical and Pharmaceutical Research Unit and ${ }^{4}$ College of Medicine, QU Health, Qatar University, Doha, Qatar

\section{Background}

- Colorectal cancer (CRC) is the third most common type of cancer in the world and its incidence is increasing constantly.

- In 2018, CRC cases reached 1.85 million with more than 880,000 deaths worldwide.

- Current chemotherapies for CRC, including 5fluorouracil (5-FU), are not efficient with severe side effects like neutropenia, bone marrow suppression and renal dysfunction. More importantly, tumors tend to develop resistance against these drugs.

- Elaeagnus Angustifolia ( $E A$ ) is a traditional plant known to possess numerous therapeutic and pharmacological properties including anti-inflammation, antioxidant and gastroprotective effects. More importantly, based on our recent investigations, EA plant extract can be used as a potential treatment against HER2-positive breast and oral cancers.

- Herein, the effect of EA extract on CRC was investigated in vitro, using KRAS CRC cell lines (HCT-116 and LoVo), and in vivo, using the Drosophila melanogaster model for $K R A S$ gene which is known to develop CRC.

\section{Objectives}

- To determine the effect of $E A$ extract in vitro using two CRC cell lines.

- To establish a new in vivo colon cancer model using $D$. melanogaster with KRAS gene mutation and use it to explore the effect of $E A$ extract on their survival.

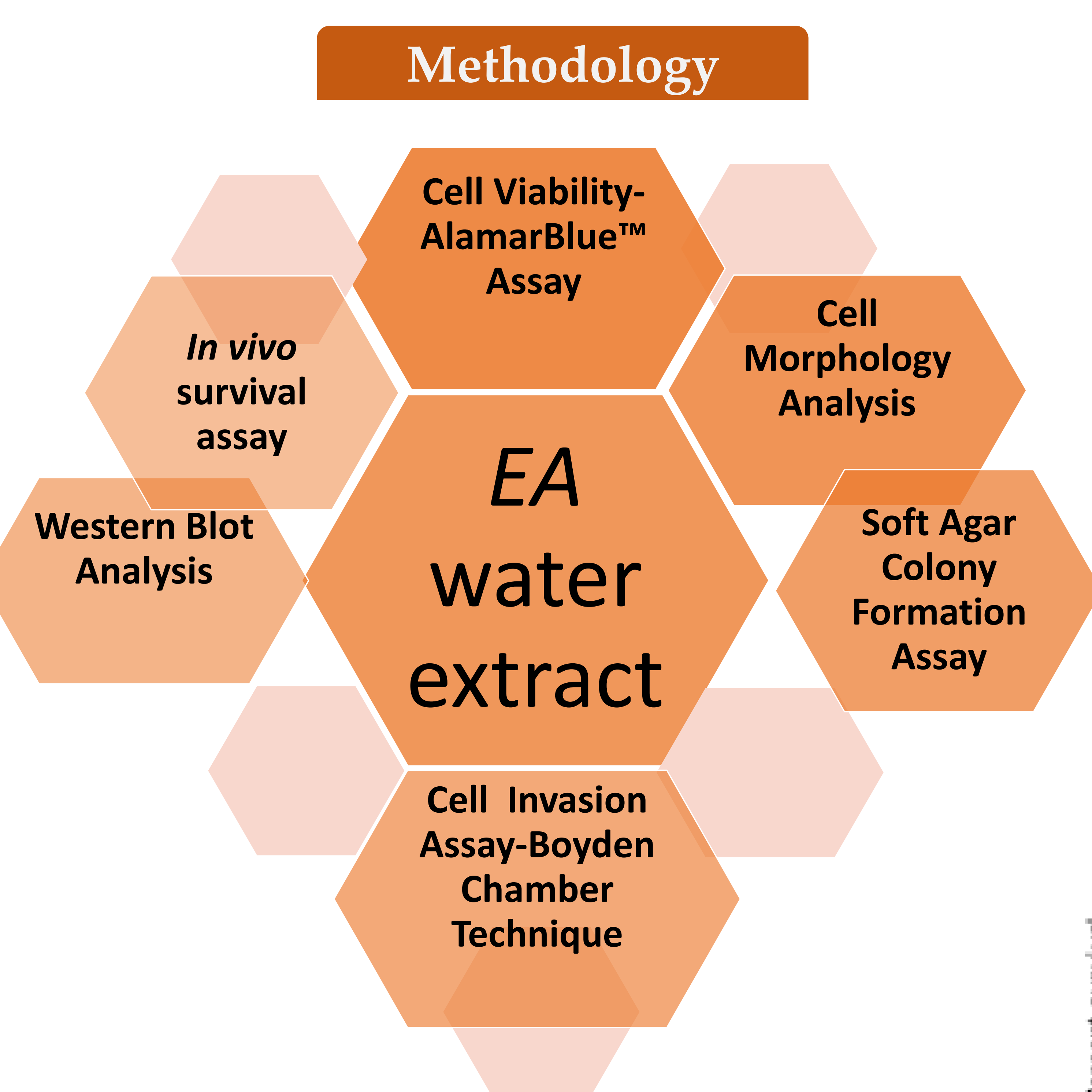

\section{Conclusion}

- EA extract has antiproliferative effect against CRC and its molecular pathway. Via the inhibition of EGFR and AKT in both CRC cell lines. Furthermore, the downregulation of Vimentin and increased expression of E-cadherin decreases cell motility and invasion ability of CRC cells by reversing EMT.

- EA increases the survival rate of both transgenic and wild type strains of D. melanogaster.

- Further studies are needed to prove the anticancer activity of EA against CRC.

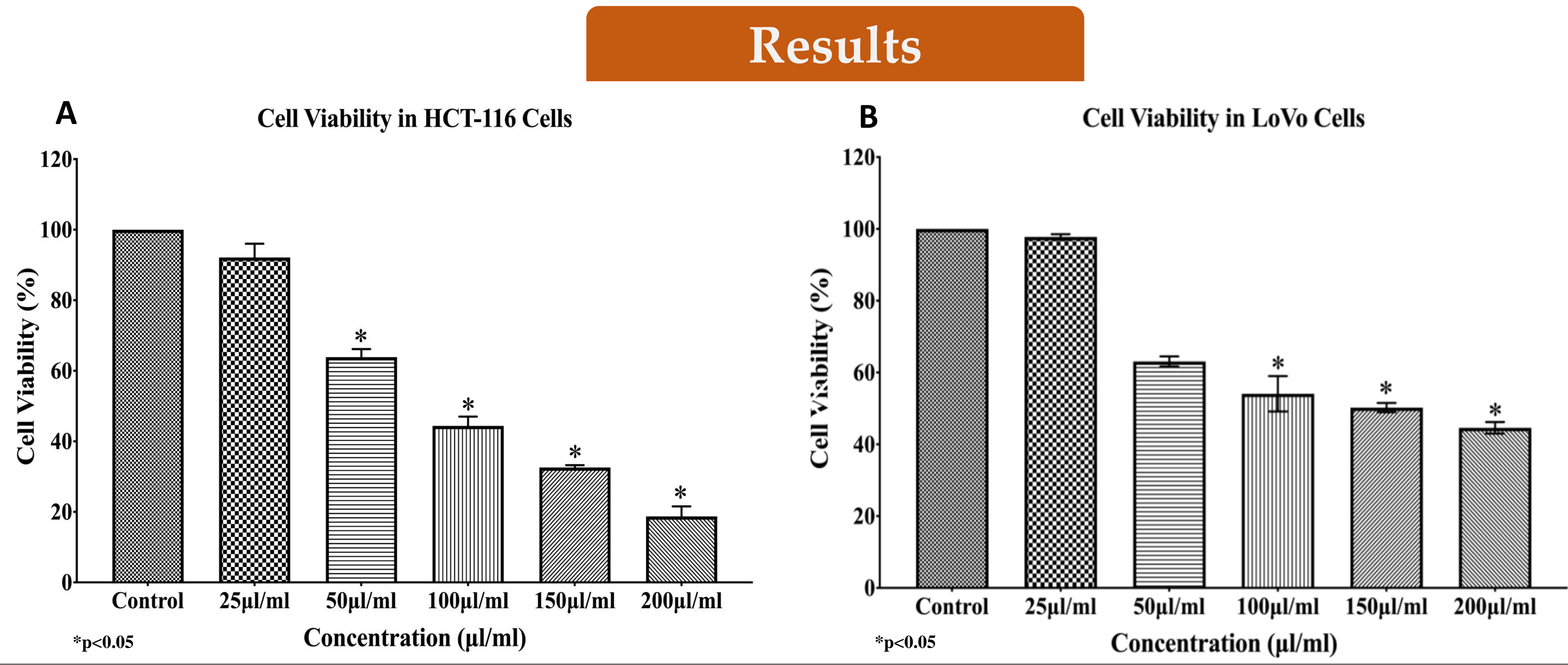

Figure 1 (A and B): Effects of EA plant extract on cell viability of CRC cells lines (A). HCT-116 and (B). LoVo at $48 \mathrm{~h}$.

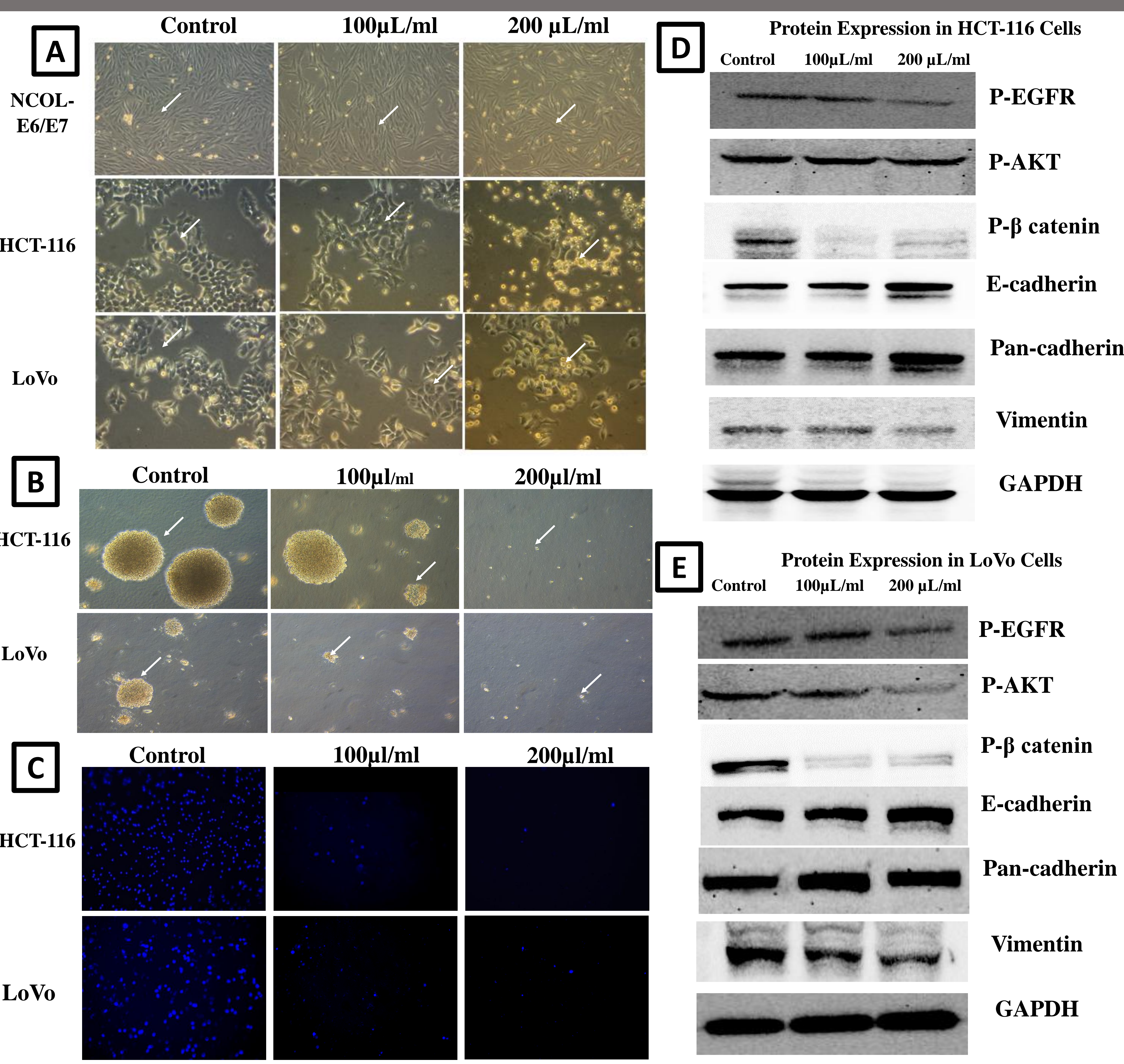

Figure 2 (A, B, C,D, and E): (A).Effects of EA plant extract onCRC morphology after 48 hours. (B) Effect of EA extract on colony formation in CRC cell lines. (C) Effect of EA flower extract on cell invasion of CRC cells. (D and E) Effect of EA extract on EMT biomarkers.

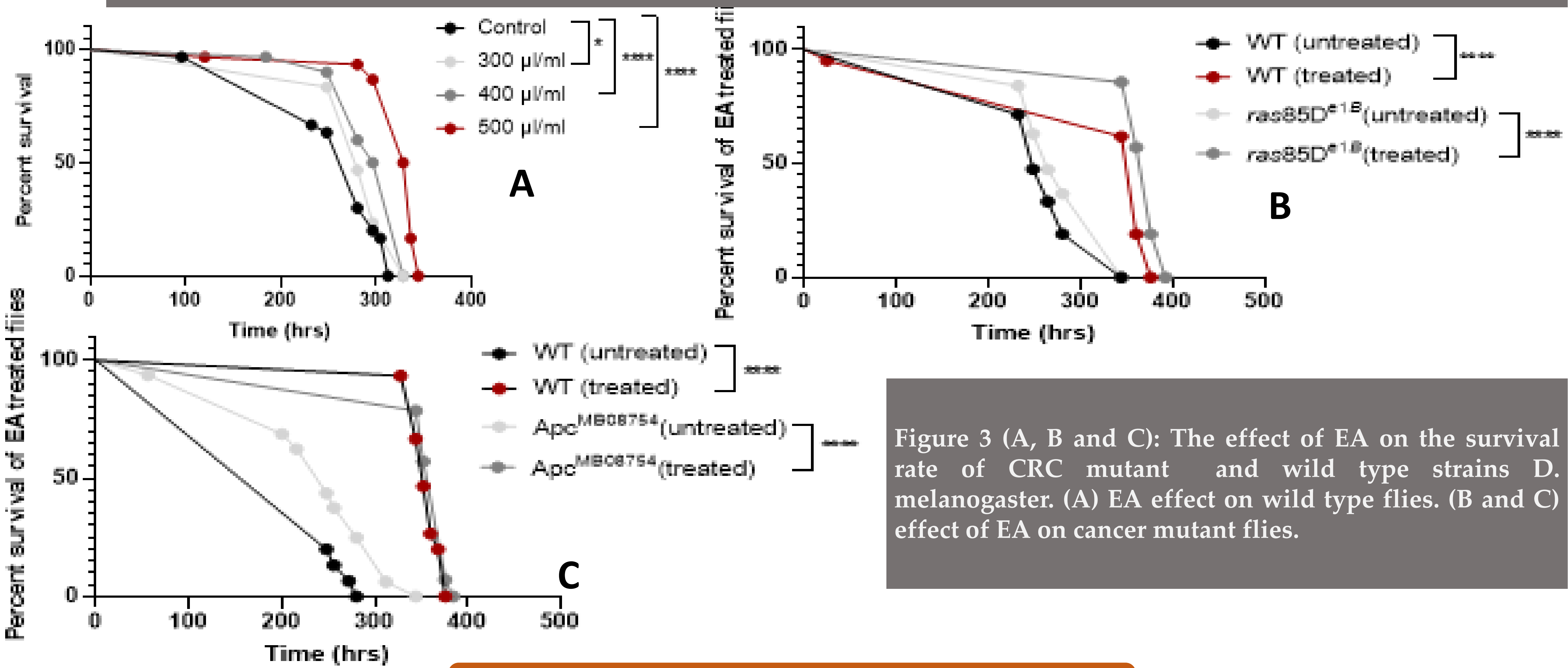

Acknowledgment

This work was supported by grants \#QUST-1-CPH-2021-22 and QUCG-CPH-20/21-4 from Qatar University. 